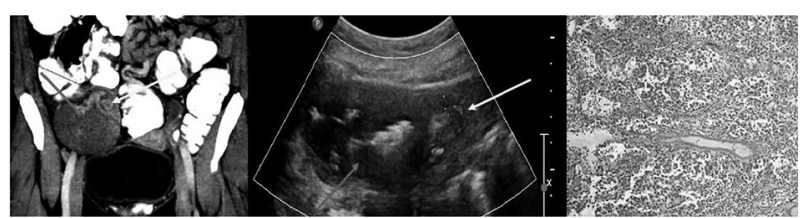

Abstract 14 Figure 1 Testicular Seminoma - From left to right coronal reconstruction of CT scan and US images depicting RIF mass in red arrow and twisted pedicle in yellow arrow. Histopathology image demonstrate malignant cell proliferation of classical seminomatous noncohesive, large, round \& atypical cells with atypical nuclei owing prominent nucleoli \& granular cytoplasm in right gonad

circumference fallopian tube and fimbria were identified bilaterally. Because of bilateral orchidectomy with normal male karyotype and normal male secondary sexual characters, regular testosterone hormone replacement therapy (testosterone $1000 \mathrm{mg} /$ every 3 month) was instituted.

Conclusion Patients with persistent Mullerian duct syndrome or internal male pseudohermaphroditism have 46 XY karyotype, near normal development of secondary sexual characters and persistent Mullerian duct structures i.e., uterus, cervix, fallopian tubes and proximal vagina. Our patient was diagnosed very late possibly due to cultural issues and stigmata attached to pseudohermaphroditism.

\section{OVARIAN GERM CELL TUMOURS; EXPERIENCE OF A REGIONAL CENTRE}

${ }^{1}$ Douglas Cartwright, ${ }^{2}$ Ashita Waterston, ${ }^{2}$ Jeff White. ${ }^{1}$ NHS Greater Glasgow and Clyde; ${ }^{2}$ Beatson West of Scotland Cancer Centre

\subsection{6/postgradmedj-2018-fpm.26}

Introduction Ovarian germ cell tumours are rare but aggressive cancers which have histological and treatment similarities with the more common male germ cell tumours. However the outcomes are poorer for women with germ cell tumours than for men. ${ }^{1}$ Historically, in the west of Scotland, female germ cells patients were managed within standard gynaecological cancer services and male patients in a dedicated germ cell unit. Due to the highly specialised nature of the condition and the experience of the clinicians using similar chemotherapy regimens, the management of ovarian germ cells was incorporated into the germ cell unit. We present the experiences of this unit over the last 6 years.

Method We reviewed data from all women with ovarian germ cell tumours treated or followed up under the regional germ cell unit. Fertility is an important consideration for these young women and we investigated the rate of fertility sparing surgery, utilisation of pre-chemotherapy fertility treatments and the rate of successful pregnancies. With the Introduction of the Cisplatin, Vincristine, Methotrexate, Bleomycin with alternate Actinomycin, Cyclophosphamide and Etoposide chemotherapy regimen (POMB/ACE) there was a concern that this will cause problems with monitoring during treatment as it associated with elevation of alpha fetoprotein (AFP) levels which is used as a marker or response. We compared AFP levels between $\mathrm{POMB} / \mathrm{ACE}$ and the bleomycin, etoposide, cisplatin (BEP) regimen used in the adjuvant or neo-adjuvant setting.

Results 26 patients have been treated or followed up by the unit over the last 6 years. Median follow up is 55 months (range 3 months - 31 years). The all-cause mortality is 0 and the relapse rate is $19 \%$. Relapses were spread equally through the treatment groups. 21 out of 24 eligible patients received fertility sparing surgery. Of these there has been 6 successful pregnancies following treatment.

There was no significant difference in AFP levels between BEP and POMB/ACE groups.

Conclusions Outcomes for ovarian germ cell patients in the west of Scotland are comparable to published case series. The low incidence makes it difficult to identify statistically significant differences however we hope to promote collaboration and gather more data through a national ovarian germ cell database set up through the Scottish Germ Cell Group.

\section{REFERENCE}

1. Murugaesu N, Schmid P, Dancey G, Agarwal R, Holden L, McNeish I, et al. Malignant ovarian germ cell tumors: Identification of novel prognostic markers and long-term outcome after multimodality treatment. I Clin Oncol 2006;24:4862-6.

\section{A CASE OF PYREXIA OF UNKNOWN ORIGIN AND RECURRENT HOSPITAL ADMISSIONS IN A CARDIAC PATIENT}

${ }^{1}$ Syed Emir Irfan Wafa, ${ }^{2}$ Raheel Ahmed, ${ }^{3}$ Kay Teck Ling, ${ }^{3}$ Peter Carey. ${ }^{1}$ University Hospitals of Leicester NHS Trust; ${ }^{2}$ University Hospitals of North Midlands NHS Trust; ${ }^{3}$ University Hospitals of Derby and Burton NHS Foundation Trust

\subsection{6/postgradmedj-2018-fpm.27}

Introduction Pyrexia of unknown origin (PUO) is defined as a temperature greater than $38.3^{\circ} \mathrm{C}$ on several occasions, accompanied by three weeks of illness and a failure to reach a diagnosis after one week of inpatient investigations. ${ }^{1}$ We describe a case of a 72-year-old man with a significant cardiac history and a pacemaker in-situ presented to the emergency department of a district general hospital with sepsis five days after he had his pacemaker unit batteries changed. $\mathrm{He}$ had deranged vital signs, productive cough and pyrexia. He had a normal chest plain radiograph (CXR). However, with right basal crackles on auscultation, he was empirically treated for a lower respiratory tract infection (LRTI) with intravenous antibiotics. The patient was discharged but had to be readmitted four times over the span of 4 months due to recurrent pyrexia. Repeated Trans-thoracic (TTE) and Trans-oesophageal (TOE) echocardiograms, and CT neck/thorax/abdomen/pelvis were done however no evidence of infection was found on the scans. A FDG-PET scan [figure 1] was eventually done which showed evidence of pacemaker-lead infection. His pacemaker unit was removed and replaced with a REVEAL device. He improved clinically with no further hospital admission to date.

Methods Routine blood test only showed raised WCC and CRP. Connective tissue disease screen, viral and tumour
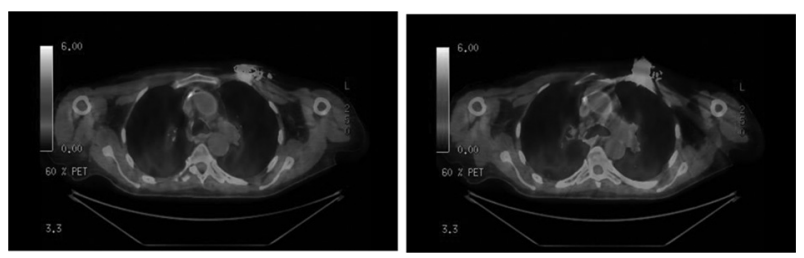

Abstract 16 Figure 1 FDG-PET scan images showing increased focal activity around pacemaker leads 


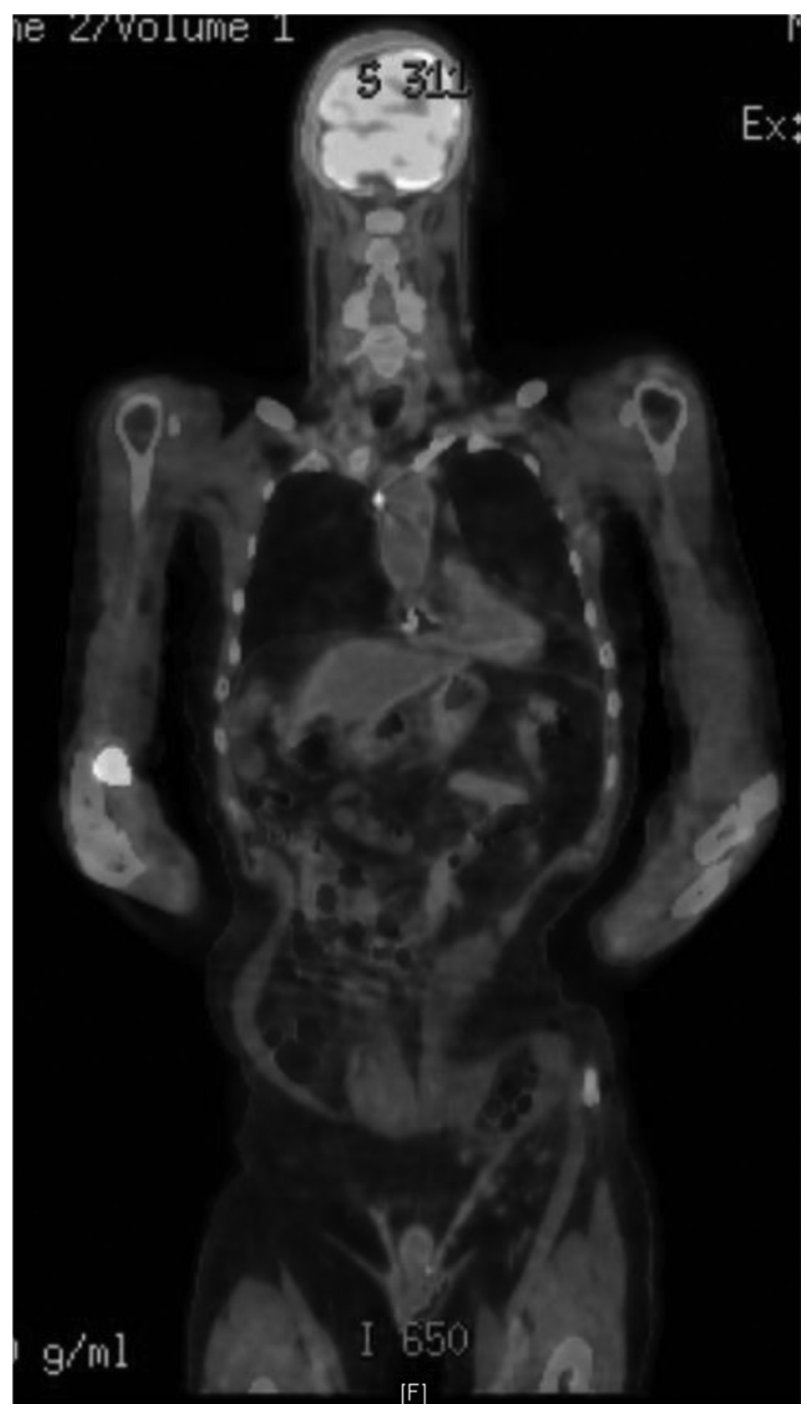

Abstract 16 Figure 2 CT appearances: no evidence of soft tissue abnormality. There is a small focus of increased activity (SUV-max 7) around the pacemaker leads as they pass from the left brachiocephalic vein into the superior vena cava. Increased activity in the right antecubital fossa correlates to some tissuing at the injection site

markers, syphilis serology, and hepatitis screen were negative. Two out of six blood cultures (on separate admissions) done grew Enterobacter cloacae. Repeated CXRs, CT scans and Echocardiograms showed no evidence of collection/infection. A FDG-PET scan was carried out which detected a focus point of increased activity around the pacemaker leads.

Results Enterobacter cloacae is a nosocomial, gram-negative, facultatively anaerobic, rod-shaped bacterium from the Enterobacteriaceae family. It is usually found in the gut normal flora and skin of humans. It can colonise surgical equipment, operative cleaning solutions and heparin solution used to irrigate certain intravascular devices. ${ }^{2}$ The surgical equipment used in this patient's pacemaker battery change may have been colonised with the bacteria which could explain why it was present in the blood cultures. Another explanation would be that it was a skin commensal for the patient. However, to our knowledge, no other cases from the hospitals involved have been identified or reported recently. Unfortunately, when the pacemaker was removed in our patient, the pacemaker leads were not sent for culture and were discarded. The type of
PET scan used in this case was the FDG- PET. Hybrid F18FDG-PET/CT has high sensitivity and relative non-specificity for malignancy, infection and inflammation. ${ }^{3} 4$ The subsequent removal of the pacemaker led to the improvement and resolution of symptoms.

Conclusions This case has highlighted the importance to explore previous hospital admissions, building a timeline of events, which could be leading to the patients' current presentation. Moreover, patients presenting with pyrexia after a recent prosthetic implantation should be investigated for prosthesis-led-infections, despite negative investigation results. Therefore, the approach to investigate and treat known source of infections and pyrexia of unknown origin needs to be done systematically according to set hospital protocols and evidencebased medicine.

\section{REFERENCES}

1. Petersdorf RG, Beeson PB. Fever of unexplained origin: report on 100 cases Medicine (Baltimore) 1961;40:1-30.

2. Mezzatesta ML, Gona F, Stefani S. Enterobacter cloacae complex: clinical impact and emerging antibiotic resistance. Future Microbiol 2012 July;7(7):887-902.

3. Hao $R$, Yuan $L$, Kan $Y$, et al. Diagnostic performance of $18 F-F D G ~ P E T / C T$ in patients with fever of unknown origin: a meta-analysis. Nucl Med Commun 2013;34(7):682-8

4. Balink $H$, Verberne $H J$, Bennink RJ, et al. A rationale for the use of F18-FDG PET/ $\mathrm{CT}$ in fever and inflammation of unknown origin. Int I $\mathrm{Mol}$ Imaging 2012;2012:165080. doi:10.1155/2012/165080

\section{ESTABLISHING A PHYSIOLOGICALLY RELEVANT IN VITRO MODEL FOR ISCHAEMIC STROKE INJURY IN BRAIN ENDOTHELIAL CELLS}

Gabriela Warpsinski, Salil Srivastava, Thomas P Keeley, Paul Fraser, Giovanni E Mann. King's College London

\subsection{6/postgradmedj-2018-fpm.28}

Introduction Ischaemic stroke is a major contributor to adult disability in developed countries. However, the only clinically approved acute treatment is delivery of the thrombolytic agent, alteplase, that has a narrow window of 6 hour poststroke. To better screen treatments for ischaemic stroke, a more relevant in vitro model for ischaemic stroke is needed. Numerous studies have demonstrated that brain microvascular endothelial cells experience an $\mathrm{O} 2$ environment ranging from 4-7 $\mathrm{kPa}$, on the contrary most cell culture studies are conducted under ambient $\mathrm{O} 2$ levels, $18 \mathrm{kPa}$ that inevitably expose cells to hyperoxia. To better understand the consequences of $\mathrm{O} 2$ levels, mouse brain endothelial cell line, bEnd.3, was cultured long-term under $18 \mathrm{kPa}$ or $5 \mathrm{kPa} \mathrm{O} 2$ levels, and cell sensitivity to in vitro model of ischaemic stroke was assessed.

Methods bEnd. 3 cells were cultured long term (5 days) under $18 \mathrm{kPa}$ or $5 \mathrm{kPa} \mathrm{O} 2$ levels and subjected to oxygen-glucose deprivation (OGD) for 2-12 hour. Cell viability was then assessed using ethidium bromide staining. Intracellular $\mathrm{O} 2$ content was also measured using an $\mathrm{O} 2$ sensitive nanoparticle probe, MitoXpress-Intra $(2.5 \mu \mathrm{g} / \mathrm{ml})$, in an O2-regulated plate reader from BMG Labtech. Statistical analysis was performed using student's t-test or two-way analysis of variance (ANOVA) followed by Bonferroni Post Hoc test.

Results In the present study, the bEnd.3 cell phenotype was determined following long-term (5 days) culture under $18 \mathrm{kPa}$ or $5 \mathrm{kPa} \mathrm{O} 2$. Under $5 \mathrm{kPa} \mathrm{O}$, the intracellular $\mathrm{O} 2$ content of bEnd.3 cells was approximately $3.6 \mathrm{kPa} \mathrm{O} 2(\mathrm{n}=3)$ and this 